\title{
Mathematical Model for Safety Score Calculation for Validation of Coach Operators in the UK
}

\author{
Manoharan Ramachandran, Reza Sahandi, Simant Prakoonwit, Wajid Khan \\ Faculty of Science and Technology, Bournemouth University, \\ Bournemouth, United Kingdom \\ \{mramchandran, rsahandi, sprakoonwit, wkhan\} @bournemouth.ac.uk
}

\begin{abstract}
Coaches are considered the safest mode of road transport for school trips. In the last decade alone, 1191 children were injured in 371 coach crashes in the UK. Consequently, the UK government enforced strict regulations on coach operators to reduce accidents. During 2016, 137 coach operator licenses have been revoked due to operator non-compliance. To increase safety of children travelling by coaches, we previously proposed a safety transport model for validation of coach operators. In this paper, a mathematical model for calculation of safety scores is presented. Real data from two transport organisations was used to test the model. Results show that, the proposed mathematical model works very well, as illustrated in this paper.
\end{abstract}

Keywords: Safety score calculation; coach operator validation; school journeys; coach transport.

\section{Introduction}

Coaches are considered as the safest mode of school transport for children [1]. Every year, in England alone more than 48000 school trips are made [2] and for most of the trips, schools rely on coach operators [3]. Analysis of the national accidents data in the UK revealed that, in the last 10 years, 1191 children have been injured in 371 coach crashes [4]. Driver errors and faults in the vehicles due to operators' noncompliance were reported as the major contributory factors for these coach crashes [5]. To reduce coach accidents, the UK government has created strict regulations to be applied by coach operators [6]. The UK government has also developed a coach Operator Compliance Risk Score (OCRS) system [7]. The system calculates the compliance risk scores for all the operators in the UK based on their fleet and drivers performance in last three years. If during an inspection a vehicle or a driver is found to be non-compliance to the safety rules, the operator will be referred for a public enquiry. An operator may lose its license if found guilty in a public enquiry. But OCRS only applies to the operators and not for their individual vehicles or drivers. Increasingly a number of operators are losing their licenses every year. In 2016 alone, 137 coach operators' licenses have been revoked in the UK, due to their noncompliance [8]. Accidents are still happening despite having strict regulations. This raises the question whether school transport through coaches in the UK is really safe? In a survey conducted in the Luton Borough Council in the UK, it was found that 
coaches are booked for trips based on trust of Coach Operators and schools rarely check the operators for their compliance with the safety regulations [9]. A safety transport model for schools which provides safety scores for validation of coach operators in the UK has been developed [10]. This will enable schools to check safety scores of coach operators, their vehicles and drivers before booking coaches for the journeys. This paper provides details of a mathematical model for calculation of safety scores, which are used in the model. The remainder of this paper is organized as follows. A short description of our safety model is provided in section 2 . The mathematical model is presented in section 3 , followed by safety scores calculation in section 4 and testing the model in section 5. Finally, conclusions and future works are outlined in section 6 .

\section{Coach Operator Validation Model}

The proposed model consists of 5 steps as shown in Figure 1. In step 1, coach operator's data which includes vehicle data (such as, safety checks, MOT, insurance, etc.) and driver's data (such as, points on the license and its expiry date, DBS checks, experience etc.) along with Operator Compliance Risk (OCR) score are obtained. In step 2, the data is verified through a comparison process with an authorized database. Step 3 assigns weights to the data parameters based on the UK government's scoring system [7]. In step 4 safety scores are calculated. Finally, in step 5, the safety scores are presented along with the prices obtained from a quote engine. This paper focuses on the steps 3 and 4 of the process which gives a brief description of the validation process of coach operators. This also includes detailed discussions on the calculation of safety scores step 4 in the validation process.

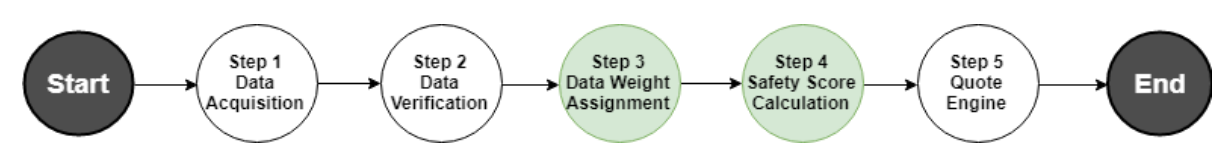

Fig. 1. Cloud based coach journey validator model

\section{Mathematical Model}

Figure 2 illustrates the process and assignment of weights for the calculation of the safety scores. The calculation starts from the assignment of weight $(w)$ to each parameter. 


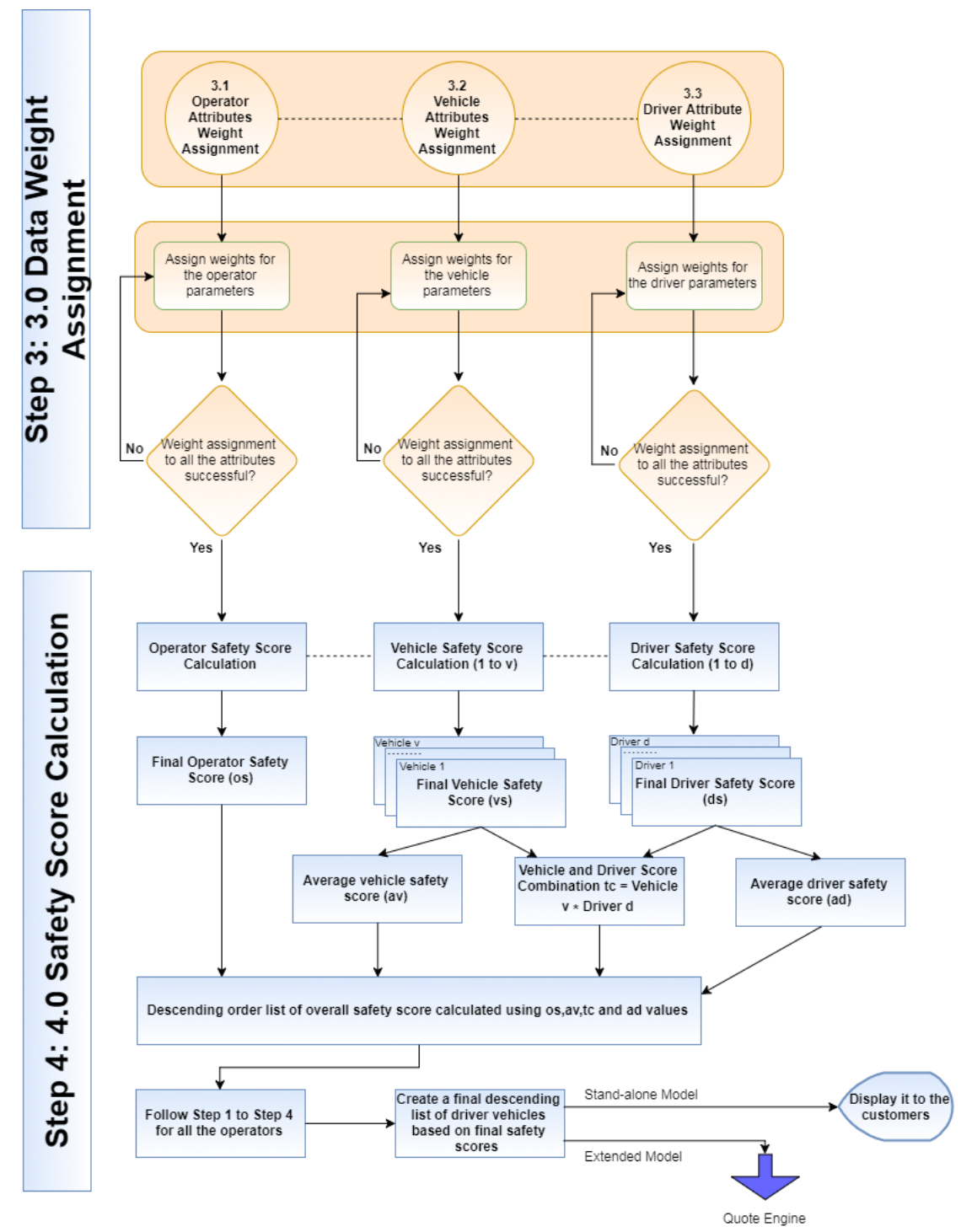

Fig. 2. Data weight assignment and safety score calculation.

Coach operator's data comprises of attributes $\left(a_{n}\right)$ and parameters $\left(p_{m}\right)$ where $n$ denotes the total number of attributes and $m$ denotes the total number of parameters for the attributes (Eg. OCRS is an operator's attribute: green, amber, red and grey are the OCRS parameters). An attribute $\left(a_{1}\right)$ may have more than one parameters ranging from $p_{1}, p_{2}, \ldots, p_{b}$ and $p_{m}$ where $p_{b}$ denotes the not applicable parameter which is necessary to exclude the attributes which are not applicable to an operator at a particular time. 
Weights for the parameters for the operator, their vehicles and drivers are then assigned. The weights are assigned based on the UK government's scoring system [7]. In this respect $x_{i}$ denotes the total weight for non-applicable attributes, $y_{i}$ the total weight possible when all the attributes have maximum weights and $z_{i}$ the overall weight obtained for all the attributes. The following calculations are based on one operator, its vehicles and drivers. The same formulae can be used to calculate the safety scores of all the operators which are explained in Section 4. The individual safety scores for the operator $(o s)$, its vehicles $(v s)$ and drivers $(d s)$ are then calculated

Equation (1) shows the calculation of the overall safety score for an operator, where $n$ denotes the total number of operator attributes, $z_{i}$ the total weight obtained by all the attributes, $y_{i}$ denotes the total weight possible when all the attributes maximum weights and $x_{i}$ denotes the total weight for non-applicable attributes.

$$
o s=\left(\frac{\sum_{i=1}^{n} z_{i}}{\sum_{i=1}^{n} y_{i}-\sum_{i=1}^{n} x_{i}}\right) \times 100
$$

Equation (2) shows the calculation of vehicle's safety score, where $n$ denotes the total number of vehicle attributes. Other parameters are similar to Equation (1),

$$
v s=\left(\frac{\sum_{i=1}^{n} z_{i}}{\sum_{i=1}^{n} y_{i}-\sum_{i=1}^{n} x_{i}}\right) \times 100
$$

Equation (3) shows the calculation of driver's safety score, where $n$ denotes the total number of driver attributes. Other parameters are similar to Equation (1),

$$
d s=\left(\frac{\sum_{i=1}^{n} z_{i}}{\sum_{i=1}^{n} y_{i}-\sum_{i=1}^{n} x_{i}}\right) \times 100
$$

All the safety scores (os, $v s$ and $d s$ ) are expressed out of 100 (percentage). One operator may have more than one vehicle and a driver. In Equation (4), av is the average safety scores for all the vehicles and $u$ denotes the total number of vehicles belongs to an operator and $v s_{i}$ the safety score for vehicle $i$ respectively.

$$
a v=\left(\frac{1}{u}\right) \times \sum_{i=1}^{u} v s_{i}
$$

In Equation (5), $a d$ is the average safety scores for the drivers of an operator, $e$ denotes the total number of drivers belongs to the operator, $d s_{i}$ denotes the safety score for driver $i$.

$$
a d=\left(\frac{1}{e}\right) \times \sum_{i=1}^{e} d s_{i}
$$

Average scores for a vehicle and driver(s) are useful information for recommendation of operators to a customer, as well denoting the safety level of the entire fleet. 


\section{Safety Score Calculation for a Journey}

To calculate the safety score for a journey, safety score combinations of available vehicles and drivers in a fleet are used. To find the best possible driver \& vehicle combinations the steps below are followed;

Step 1: The number of possible vehicle and driver combination $(c)$ are calculated using Equation (6). In this equation, $u$ and $e$ denote the total number of vehicles and drivers respectively.

$$
c=u * e
$$

Step 2: To find the sample space $\Omega$ between the vehicle's safety scores and driver's safety scores, Equation (7) is used. In this equation, $v s$ and $d s$ denote vehicle and driver safety scores respectively.

$$
\text { Sample Space, } \Omega=\left\{\left(v s_{1}, d s_{1}\right),\left(v s_{2}, d s_{2}\right), \ldots\left(v s_{u}, d s_{e}\right)\right\}
$$

Step 3: To find the sum for all the combinations, Equations (8) is used. To find the average for individual combinations of $q$, Equation (9) is used.

$$
\begin{gathered}
q=\text { Individual Sum }\{\Omega\} \\
q=\left\{v d_{1}, v d_{2}, \ldots \ldots \ldots, v d_{c}\right\}
\end{gathered}
$$

Where, $v d_{1}=v s_{1}+d s_{1}, v d_{2}=v s_{1}+d s_{2}, \ldots v d_{c}=v s_{u}+d s_{e}\left(v s_{u}+d s_{e}\right.$ denotes the last possible driver and vehicle combination)

$$
\operatorname{avg}=q *\left(\frac{1}{2}\right)
$$

Step 4: To arrange the combinations in descending order, Equation (10) is used.

$$
l[i]=\operatorname{sortdesc}(\operatorname{avg})
$$

$l$ is the list of vehicle-driver combination averages in descending order and $i$ represents the individual values inside the $l$ where, $i=1$ to $c$.

$$
f l[i]=(o s * \mu) / 100+(a v * \alpha) / 100+(a d * \beta) / 100+(l[i] * \rho) / 100
$$

Equation (11) shows the final safety score list $(f l[i])$ for one operator. To give weightage for the values of $o s, a v, a d$ and $l[i]$, constants $(\mu, \alpha, \beta, \rho)$ are used. By using these constants, weights for individual variables can be specified (ex. $\mu=10, \alpha=5, \beta=5, \rho=80$ ). Using the Equation (12), list of possible driver and vehicle combinations under an operator who is registered with the coach broker can be calculated. The final list of operators and their safety scores will be listed as,

$$
j s=\operatorname{sortdesc}\left(f l_{1}[i], f l_{2}[i], f l_{3}[i] \ldots, f l_{\sigma}[i]\right)
$$

Where, $\sigma$ denotes the total number of operators registered with a coach broker, $i$ denotes the number of vehicle and driver combinations for each operator which ranges from 1 to $c$ and $j s$ denotes the Journey Score list. The total number of 
combinational values inside the $j s$ can be calculated by adding $c$ values of all the operators (i.e) $j s\left[c_{1}+c_{2}+. .+c_{\sigma}\right]$, where $\sigma$ denotes the total number of operators registered with the coach broker.

\section{Testing}

The proposed equations were tested for appropriateness and accuracy using real data from two coach operators in Luton in the UK who are registered with the Luton Borough Council. For confidentiality, the names of the operators are anonymised as Operator A and B. Operator A had 3 coaches and 4 drivers. Operator B had 2 coaches and 3 drivers. Following the information from previous section weights for the operator and the parameters for its vehicles and drivers were assigned and using the Equation 1 and 2 the values were calculated and recorded. To obtain the scores for A: Overall safety score - Equation (1),

$$
\text { os }=\left(\frac{8}{11-1}\right) \times 100=>\text { os }=80 \%
$$

Individual vehicle safety score - Equation (2),

$$
v s_{1}=\left(\frac{8}{12-2}\right) \times 100=>v s_{1}=80 \%
$$

Repeating the above equation and by applying the operator parameters for all the vehicles, following values are obtained; $v s_{2}=81.81 \% v s_{3}=81.81 \%$.

Average vehicle safety score - Equation (4),

$$
a v=\left(\frac{1}{3}\right) \times(80+81.8+81.81)=>a v=81.21 \%
$$

Similarly to calculate the driver safety scores:

Individual driver safety score - Equation (3),

$$
d s_{1}=\left(\frac{7}{10-0}\right) \times 100=>d s_{1}=70 \%
$$

Repeating the above equation for all the 4 drivers, $d s_{2}=55.55 \% ; d s_{3}=90.00 \%$ and $d s_{4}=60.00 \%$.

Average driver safety score - Equation (5),

$$
a d=\left(\frac{1}{4}\right) \times(70+55.55+90+60)=>a d=68.88 \%
$$

Table 1 and 2 show the safety scores of the Operator A for their individual coaches and drivers. 
Table 1. Vehicle Safety score values

\begin{tabular}{ccc}
\hline $\begin{array}{c}\text { Vehicle } \\
\text { No. }\end{array}$ & $v s_{v}$ & Score \\
\hline 1 & $v s_{1}$ & $80.00 \%$ \\
2 & $v s_{2}$ & $81.81 \%$ \\
3 & $v s_{3}$ & $81.81 \%$ \\
& & $a v=81.21 \%$ \\
\hline
\end{tabular}

Table 2. Driver Safety score values

\begin{tabular}{ccc}
\hline $\begin{array}{c}\text { Driver } \\
\text { No. }\end{array}$ & $d s_{d}$ & Score \\
\hline 1 & $d s_{1}$ & $70.00 \%$ \\
2 & $d s_{2}$ & $55.55 \%$ \\
3 & $d s_{3}$ & $90.00 \%$ \\
4 & $d s_{4}$ & $60.00 \%$ \\
$e=4$ & & $a d=68.00 \%$ \\
\hline
\end{tabular}

To find the best possible driver \& vehicle combinations following steps are followed,

step 1: The total numbers of possible combinations, using Equation (6): $c=12$. This means, there are 12 possible driver-vehicle combinations in total.

step 2: The sample space, using Equation (7),

$$
\text { Sample Space, } \Omega=\{(80,70),(80,55.55), \ldots,(81.81,60)\} \text {. }
$$

step 3: The sum for all the combinations using Equation (8),

$$
q=\{(80+70),(80+55.55), \ldots,(81.81+60)\} .
$$

Using Equation (9)

$$
\operatorname{avg}=\{150,135.55, \ldots, 141.81\} *(1 / 2)=>\operatorname{avg}=\{75,67.77, \ldots ., 70.90\}
$$

step 4: The combinations in descending order using Equation (10):

$$
\begin{gathered}
l[i]=\operatorname{sort}-\operatorname{desc}(\{75,67.77,85,70,75.90,68.68,85.90,70.905,75.905,68.68, \\
85.90,70.90\})
\end{gathered}
$$

$l[i]=\{85.90,85.90,85,75.90,75.90,75,70.90,70.90,70,68.68,68.68,67.775\}$ For the final safety score list using Equation (11): The constant values used are: $\mu=$ $10, \alpha=5, \beta=5, \rho=80$ and $i=1$ to 12 .

$$
\begin{gathered}
f l[1]=(80 * 10) / 100+(81.21 * 5) / 100+(68 * 5) / 100+(85.90 * 80) / 100 \\
f l[1]=84.18 \%
\end{gathered}
$$

Where, $\mu=10, \alpha=5, \beta=5, \rho=80$ and $i=1$ to $12 . f l_{1}[i]=$ Final safety score combination list for 1 operator. $f l_{1}[\mathrm{i}]=\{84.18,84.18,83.46,76.18,76.18,75.46$, $72.18,72.18,71.46,70.40,70.40,69.68\}$. Table 3 shows the mapping of average values and sums for the vehicle and driver combinations (i.e complete list of all the final values for Operator A). 
Table 3. Mapping of average values with vehicle and driver combinations for Operator A

\begin{tabular}{llllll}
\hline$l[\mathrm{i}]$ & $\mathrm{vs}_{\mathrm{v}}$ & $d s_{d}$ & Sum & Average & $f l[i]$ \\
\hline$l[1]$ & $\mathrm{vs}_{2}$ & $d s_{3}$ & 171.81 & 85.90 & $84.18 \%$ \\
$l[2]$ & $\mathrm{vs}_{3}$ & $d s_{3}$ & 171.81 & 85.90 & $84.18 \%$ \\
$l[3]$ & $\mathrm{vs}_{1}$ & $d s_{3}$ & 170 & 85 & $83.46 \%$ \\
$l[4]$ & $\mathrm{vs}_{2}$ & $d s_{1}$ & 151.81 & 75.90 & $76.18 \%$ \\
$l[5]$ & $\mathrm{vs}_{3}$ & $d s_{1}$ & 151.81 & 75.90 & $76.18 \%$ \\
$l[6]$ & $\mathrm{vs}_{1}$ & $d s_{1}$ & 150 & 75 & $75.46 \%$ \\
$l[7]$ & $\mathrm{vs}_{2}$ & $d s_{4}$ & 141.81 & 70.90 & $72.18 \%$ \\
$l[8]$ & $\mathrm{vs}_{3}$ & $d s_{4}$ & 141.81 & 70.90 & $72.18 \%$ \\
$l[9]$ & $\mathrm{vs}_{1}$ & $d s_{4}$ & 140 & 70 & $71.46 \%$ \\
$l[10]$ & $\mathrm{vs}_{2}$ & $d s_{2}$ & 137.36 & 68.68 & $70.40 \%$ \\
$l[11]$ & $\mathrm{vs}_{3}$ & $d s_{2}$ & 137.36 & 68.68 & $70.40 \%$ \\
$l[12]$ & $\mathrm{vs}_{1}$ & $d s_{2}$ & 135.55 & 67.77 & $69.68 \%$ \\
\hline
\end{tabular}

The same approach is used to calculate the safety scores for Operator B. Table 4 shows the Mapping of average values with vehicle and driver combinations for Operator B

Table 4. Mapping of average values, sums for vehicle and driver combinations for Operator B

\begin{tabular}{llllll}
\hline$l[\mathrm{i}]$ & $\mathrm{vs}_{\mathrm{v}}$ & $d s_{d}$ & Sum & Average & $f l[i]$ \\
\hline$l[1]$ & $\mathrm{vs}_{2}$ & $d s_{3}$ & 171.88 & 85.94 & $84.22 \%$ \\
$l[2]$ & $\mathrm{vs}_{2}$ & $d s_{2}$ & 165.60 & 82.80 & $81.75 \%$ \\
$l[3]$ & $\mathrm{vs}_{1}$ & $d s_{3}$ & 151.64 & 75.82 & $76.12 \%$ \\
$l[4]$ & $\mathrm{vs}_{2}$ & $d s_{1}$ & 137.20 & 68.60 & $70.33 \%$ \\
$l[5]$ & $\mathrm{vs}_{1}$ & $d s_{1}$ & 135.76 & 67.88 & $69.88 \%$ \\
$l[6]$ & $\mathrm{vs}_{1}$ & $d s_{2}$ & 133.48 & 66.74 & $68.75 \%$ \\
\hline
\end{tabular}

Equation (12) can then be used to sort in descending order, the final list of vehicle/driver combinations for both the operators.

$$
\text { jsl }\left[\mathrm{c}_{\sigma=1 \text { to } 2}\right]=(84.22,84.18,84.18,83.46,81.75,76.18,76.18,76.12,75.46,72.18 \text {, }
$$

$72.18,71.46,70.40,70.40,70.33,69.88,69.68,68.74)$

\section{Conclusion and Future Work}

Safety of school transportation is a critical issue which should be addressed effectively. Safety in coach-based school transport in the UK is a less investigated area, compared to the other modes of transport for schools. Operator non-compliance is a major issue in the coach industry. This requires an urgent attention before more children lives are put at risk. This paper presented a mathematical model for calculation of safety scores for coach operators which is a part of a proposed safety transport model. This paper by applying real data from two coach operators illustrated that the mathematical model works well. As our future work, the model will be 
further validated by making it available to wider groups of practitioners/users for comments. The results will also be subsequently published.

\section{References}

1. O’neal, E., Ramirez, M., Cara, H., Tracy, Y., Stahlhut, M., Peek-Asa, C.: School Bus Crash Rates on Routine and. J. Sch. Health. 84, 575-580 (2014).

2. Drake, R.: Schools, pupils and their characteristics, https://www.gov.uk/government/statistics/schools-pupils-and-their-characteristicsjanuary-2016.

3. Move, D.G.: Comprehensive Study on Passenger Transport by Coach in Europe. (2016).

4. DfT: Department for Transport (Great Britan) Road Safety Data - Datasets, https://data.gov.uk/dataset/road-accidents-safety-data.

5. DfT: Department for Transport (Great Britan) : Contributory factors for reported road accidents, https://www.gov.uk/government/statistical-data-sets/ras50-contributoryfactors.

6. Department for Education: Home to school travel and transport guidance: Statutory guidance for local authorities. 1-37 (2014).

7. DVSA: Use the Operator Compliance Risk Score (OCRS) system.

8. Commissioners, T.: Traffic Commissioners' Annual Reports, https://www.gov.uk/government/collections/traffic-commissioners-annual-reports.

9. Ramachandran, M., Sahandi, R., Prakoonwit, S., Khan, W.: Intelligent Safety Transport Framework for Schools: A Review of Route Planning and Tracking Systems. 7, (2016).

10. Ramachandran, M., Sahandi, R., Prakoonwit, S., Khan, W., Mohd Selamat, S.A.: A Safety Transport Model for Validation of UK Coach Operators for School Journeys. In: Intelligent Transport Systems - From research and development to the market uptake. Springer (2017). 